\title{
The Influence of Deviations from the Maxwellian Electron Distribution on the Population densities in a low Temperature Monatomic Plasma
}

\author{
H. A. Claaßen \\ Institut für Plasmaphysik der Kernforschungsanlage Jülich GmbH, \\ ASSOZIATION EURATOM-KFA
}

(Z. Naturforsch. 30 a, 451-460 [1975] ; received December 24, 1974)

\begin{abstract}
A selfconsistent solution with respect to both free and bound electron energy states is presented for a low temperature monatomic plasma. The deviations from a Maxwellian electron distribution under the action of unbalanced resonance transitions of the plasma atoms are incorporated in analytical form using a recursion formula, which links the high energy tail of the electron distribution function to its low energy part. The unbalance of the resonance level may be due to diffusion processes and/or radiation escape, which reflect the influence of the plasma boundaries. The population densities of the bound electron energy states are numerically determined by an iteration procedure. The calculations were performed for a cesium plasma both without and with consideration of resonance level diffusion and ambipolar diffusion. As is to be expected, the effect of a disturbance of the electron distribution on the population densities increases with decreasing electron density and increasing electron temperature.
\end{abstract}

\section{Introduction}

The theory of nonequilibrium population densities in low temperature monatomic plasmas is usually treated on the basis of a Maxwellian electron distribution [see, for instance, ${ }^{1-4}$ ]. There are a few attempts to take into account departures from the Maxwellian electron distribution when calculating departures from the Boltzmann distribution of atomic energy levels due to radiation escape and particle diffusion. In these calculations the kinetic equation for the isotropic part of the electron distribution function is either numerically integrated ${ }^{5-7}$ or analytically solved for a rather limited energy range including the resonance excitation energy ${ }^{8-12}$.

The analytical solutions generally apply to situations, in which only one unbalanced electron collisional excitation process markedly influences the electron distribution. Such comparatively simple situations usually occur in low temperature hydrogen or alkali metal plasmas, where the main disturbance effect on the electron distribution results from the collisional unbalance of the resonance level. For such cases the analytical solution can be extended up to and even beyond the ionization energy of the plasma atoms without introducing a formalism, which would be too complicațed for a numerical evaluation. Moreover, as has been verified by the

Reprint requests to Dr. H. A. Claaßen, Institut für Plasmaphysik der KFA Jülich GmbH, D-5170 Jülich 1, Postfach 365. author in a previous paper ${ }^{13}$, a recursion formula exists, which links the high energy tail to the low energy part of the electron distribution function.

In the previous paper the study was aimed at the calculation of the disturbance of the electron distribution in a low temperature cesium plasma due to a nonequilibrium population density of the cesium resonance level. The basic simplifications underlying this calculation were the decoupling of the resonance level from all higher levels and the treatment of the electron density as a free parameter.

In this paper we drop the weak coupling assumption for the resonance level in order to perform a selfconsistent calculation with respect to both free and bound electron energy states in a low temperature cesium plasma.

In contrast to the weak coupling case the selfconsistent calculation does not yield an analytical solution to both the electron distribution and the resonance level population. Rather the resonance level population density and the electron density remain as parameters in the analytical solution for the electron distribution. Substitution of this solution into the collision integrals for inelastic electronatom collisions transforms the particle balance equations into a system of coupled nonlinear equations, which can be numerically solved by an iteration method. The evaluation of these equations considerably simplifies by application of the recursion formula for the high energy tail of the electron distribution function. 


\section{Model}

In our study we refer to the stationary state of a plasma, which is composed of electrons, atomic ions in their ground state, and atoms in their various excitation states.

We assume that the electron distribution is weakly anisotropic. We further assume that the deviation of the isotropic part of the electron distribution from a Maxwellian distribution is essentially determined by unbalanced electron collisional excitation and deexcitation of the resonance level. We thus presume that the electric field effect is limited to an electron temperature elevation over the common translational temperature of the heavy particles.

We limit the consideration to electron collisional and radiative excitation-deexcitation and ionizationrecombination processes as well as to resonance excitation diffusion and ambipolar diffusion. The diffusion processes are taken into account by introduction of characteristic inhomogeneity lengths as free parameters.

We assume that the plasma partially reabsorbs resonance radiation, but is completely transparent to nonresonance radiation. The reabsorption of resonance radiation is taken into account by a resonance radiation escape factor as a free parameter.
We neglect deviations of the low energy part of the electron distribution from a Maxwellian distribution as far as the integral coefficients in the Fokker-Planck term for electron-electron interactions, the rate coefficients for electronic three body and radiative recombination, and the translational energy exchange between electrons and heavy particles are concerned.

The above model assumptions are valid and consistent, if certain conditions are satisfied. These conditions are summarized in the Appendix.

\section{Governing Equations}

According to the above plasma model the nonequilibrium population densities of bound and free electron states can be derived by sole consideration of the particle balance equations in connection with the kinetic equation for the isotropic part of the electron distribution function. The electron energy balance may be added to compute the electron temperature elevation for a prescribed electric field strength or vice versa.

The governing equations have the following form:

a) kinetic equation for the isotropic part of the electron distribution *

$$
\begin{aligned}
& \frac{\nu_{--}^{*} \exp \eta}{\eta}\left[F(\eta) \gamma^{\prime}(\eta) \exp (-\eta)\right]^{\prime}+\eta_{0} Q_{-_{0}^{-}}^{-1}\left(\eta, \bar{\eta}_{1}\right)\left[\alpha_{1} \gamma\left(\eta-\bar{\eta}_{1}\right)-\gamma(\eta)\right] \\
& \quad+\left(1+\frac{\bar{\eta}_{1}}{\eta}\right) n_{0} Q_{-0}^{-1}\left(\eta+\bar{\eta}_{1}, \bar{\eta}_{1}\right) \exp \left(-\bar{\eta}_{1}\right)\left[\gamma\left(\eta+\bar{\eta}_{1}\right)-\alpha_{1} \gamma(\eta)\right]=0
\end{aligned}
$$

$Q_{-0}^{-1}\left(\eta, \bar{\eta}_{1}\right)$ and $\sqrt{8 k T_{-} / \pi m_{-}} v_{--}^{*}$ are respectively the cross section for electron collisional excitation of the resonance level and the electron-electron interaction frequency. $v_{--}^{*}$ is defined by the relation

$$
\begin{aligned}
v_{--}^{*}=2 \pi n_{-} \bar{q} \ln \Lambda_{--} \text {with } \bar{q} & =\left(\frac{e^{2}}{4 \pi \varepsilon_{0} k T_{-}}\right)^{2} \\
\text { and } \Lambda_{--} & =\frac{12 \pi}{\sqrt{n_{-}}}\left(\frac{\varepsilon_{0} k T_{-}}{e^{2}}\right)^{3 / 2}
\end{aligned}
$$

where the MKSA-system of units is used.

* The indices,,-+ i respectively denote electrons, atomic ions in their ground state, and atoms in their various excitation states. In particular, the index 0 signifies the atomic ground state. The energies of free and bound c'ectron states are normalized to the mean thermal election energy, i. e. we use the variables $\eta=\varepsilon_{-} / k T$ and $\vec{\eta}_{\mathrm{i}}=$ $\left(\varepsilon_{\mathrm{i}}-\varepsilon_{0}\right) / k T \ldots$ Dashes indicate derivatives with respect to $\eta$.
The factor

$$
F(\eta)=\operatorname{erf} \sqrt{\eta}-2 \sqrt{\frac{\eta}{\pi}} \exp (-\eta)
$$

arises from substitution of a Maxwellian electron distribution into the integral coefficients of the Fokker-Planck collision term.

Equation (1) has been formulated in terms of the normalized electron distribution function $\gamma(\eta)$ $=f_{-}(\eta) / f_{-}^{\mathrm{M}}(\eta)$ and the normalized resonance level population density $\alpha_{1}=n_{1} / n_{1}{ }^{\mathrm{B}}$ with

$$
\begin{aligned}
& f_{-}^{\mathrm{N}}(\eta)=n_{-}\left(\frac{m_{-}}{2 \pi k T_{-}}\right)^{3 / 2} \exp (-\eta) \\
& \text { and } \quad n_{1}{ }^{\mathrm{B}}=n_{0} \frac{g_{1}}{g_{0}} \exp \left(-\bar{\eta}_{1}\right) .
\end{aligned}
$$

In the form of Eq. (1) the kinetic equation expresses the balance between two competing effects: 
on the one hand electron-electron interactions, which tend to Maxwellize the electron gas, the Maxwellization being especially effective in the low energy regime, and

on the other hand atomic resonance excitation and deexcitation by electron impact, which tend to disturb the Maxwellization, if radiation escape and/ or excitation diffusion disturb the adjustment of a Boltzmann population density of the resonance level, the disturbance being especially effective in the high energy regime.

b) Particle balance equations for the various atomic energy levels

$$
\begin{array}{r}
\sum_{j<i}\left[\bar{N}_{-} \frac{g_{j}}{g_{i}} \exp \left(\bar{\eta}_{i}-\bar{\eta}_{j}\right) C_{j \rightarrow i}-\alpha_{i} \frac{A_{i \rightarrow j} \Lambda_{i \rightarrow j}}{\bar{v}-\bar{q} n_{-}^{\mathrm{s}}}\right]-\sum_{j>i}\left[\bar{N}_{-} C_{i \rightarrow j}-\alpha_{j} \frac{g_{j}}{g_{i}} \exp \left(\bar{\eta}_{i}-\bar{\eta}_{j}\right) \frac{A_{j \rightarrow i} \Lambda_{j \rightarrow i}}{\bar{v} \bar{q} n_{-}^{\mathrm{s}}}\right] \\
-\bar{N}_{-} C_{i \rightarrow+}+\bar{N}_{-}^{2} \frac{n_{-}^{\mathrm{s}}}{n_{i}{ }^{\mathrm{B}}} \int_{0}^{\infty} \eta \frac{\sigma_{\nu i}^{\bar{\nu}_{i}^{+}}\left(\eta, \bar{\eta}_{+}-\bar{\eta}_{i}\right)}{\bar{q}} \exp (-\eta) \mathrm{d} \eta-\frac{\Gamma_{i}}{v_{-} \bar{q} n_{-}^{\mathrm{s}} n_{i}{ }^{\mathrm{B}}}=0
\end{array}
$$

where $\Gamma_{i}$ denotes the diffusion rate of the atomic energy level $i . \bar{v}_{-}=\sqrt{8 k T_{-} / \pi m_{-}}$is the thermal electron velocity.

Equation (2) has been formulated in terms of the normalized particle densities $\alpha_{i}=n_{i} / n_{i}{ }^{\mathrm{B}}$ and $\bar{N}_{-}$ $=n_{-} / n_{-}^{\mathrm{S}}$ with

$$
n_{i}{ }^{\mathrm{B}}=n_{0} \frac{g_{i}}{g_{0}} \exp \left(-\bar{\eta}_{i}\right) \quad \text { and } \quad n_{-}^{\mathrm{s}}=\sqrt{n_{0} \frac{2 g_{+}}{g_{0}}\left(\frac{m_{-} k T_{-}}{2 \pi \hbar^{2}}\right)^{3 / 2} \exp \left(-\bar{\eta}_{+}\right)} .
$$

$g_{i}$ and $g_{+}$are the statistical weigths of the excited atoms and the ions.

The normalized collision integrals for electron collisional excitation-deexcitation and ionization-recombination processes are defined by the expressions

$$
\begin{gathered}
C_{i \rightarrow j}=\int_{\eta_{j}-\bar{\eta}_{i}}^{\infty}\left[\alpha_{i} \gamma(\eta)-\alpha_{j} \gamma\left(\eta-\bar{\eta}_{j}+\bar{\eta}_{i}\right)\right] \eta \frac{Q_{-i}^{-j}\left(\eta, \bar{\eta}_{j}-\bar{\eta}_{i}\right)}{\bar{q}} \exp (-\eta) \mathrm{d} \eta \\
C_{i \rightarrow+}=\int_{\bar{\eta}_{+}-\bar{\eta}_{j}}^{\infty}\left[\alpha_{i} \gamma(\eta)-\bar{N}_{-}^{2}\right] \eta \frac{Q_{-i}^{-\hat{\uparrow}^{-}}\left(\eta, \bar{\eta}_{+}-\bar{\eta}_{i}\right)}{\bar{q}} \exp (-\eta) \mathrm{d} \eta
\end{gathered}
$$

where $Q_{-i}^{-j}\left(\eta, \bar{\eta}_{j}-\bar{\eta}_{i}\right)$ and $Q_{-i}^{-\bar{\uparrow}^{+}}\left(\eta, \bar{\eta}_{+}-\bar{\eta}_{i}\right)$ are respectively the excitation and ionization cross sections of the atomic energy level $i$.

The radiation processes are characterized by the Einstein- $A_{i \rightarrow j}$-coefficients for spontaneous emission of line radiation reduced by the escape factors $\Lambda_{i \rightarrow j}$, and the radiative recombination cross sections $\sigma_{\nu i}^{-+}\left(\eta, \bar{\eta}_{+}-\bar{\eta}_{i}\right)$.

The diffusion processes have been taken into account by the order of magnitude relation

$$
\Gamma_{i}=0\left[\frac{D_{i} n_{i}{ }^{\mathrm{B}}}{l_{i}{ }^{2}} \alpha_{i}\right]
$$

where $D_{i}=\sqrt{\pi k T_{\sigma} / 8 m_{\sigma}} \bar{\lambda}_{i}$ and $l_{i}$ are respectively the diffusion coefficient and the characteristic inhomogeneity length of the atomic energie level $i$. $\bar{\lambda}_{i}$ is the mean free path for the excited atoms. The negative sign of the diffusion term in Eq. (2) has been chosen to allow for diffusion losses. In order to satisfy the steady state condition for the plasma as a whole, it is implicitely assumed that diffusion losses of atomic excitation levels and free electrons are compensated by a diffusion gain in the number density of the ground state atoms.

Equation (2) describes the balance between all electron collisional and radiative transitions from and into the atomic energy level $i$. In particular, the terms in the first bracket denote electron collisional and radiative excitation and deexcitation of level $i$ from and into levels $j<i$. The second bracket contains corresponding terms for excitation and deexcitation of levels $j>i$ from and into level $i$. The next two terms determine electron collisional and radiative ionization and recombination from and into level $i$. The last term describes the diffusion loss of level $i$. 
The population densities of the atomic excitation levels are again determined by two competing effects:

on the one hand electron collisional excitationdeexcitation and ionization-three body recombination processes, which tend to establish a BoltzmannSaha distribution of the atomic energy levels at the electron temperature, if the free electrons are Max- wellized, these processes being especially effective in the upper part of the level scheme, and

on the other hand radiation escape and diffusion losses, which tend to disturb the adjustment of a Boltzmann-Saha distribution, these processes being especially effective in the lower part of the level scheme.

In accordance with Eq. (2) the particle balance equation for the free electrons can be written in the form

$$
\begin{aligned}
& \bar{N}_{-} \sum_{i<i_{\mathrm{T}}} \frac{g_{i}}{g_{0}} \exp \left(-\bar{\eta}_{i}\right) C_{i \rightarrow+}-\bar{N}_{-}^{2} \frac{n_{-}^{\mathrm{S}}}{n_{0}} \sum_{i<i_{\mathrm{T}}} \int_{0}^{\infty} \eta \frac{\sigma_{\nu i}^{-+}\left(\eta, \bar{\eta}_{+}-\bar{\eta}_{i}\right)}{\bar{q}} \exp (-\eta) \mathrm{d} \eta \\
& \quad+\left.\bar{N}_{-} \sum_{i<i_{\mathrm{T}} \leqq j} \frac{g_{i}}{g_{0}} \exp \left(-\bar{\eta}_{i}\right) C_{i \rightarrow j}\right|_{\alpha_{j}=\bar{N}_{-}=}-\bar{N}_{-}^{2} \sum_{i<i_{\mathrm{T}} \leqq j} \frac{g_{j}}{g_{0}} \exp \left(-\bar{\eta}_{j}\right) \frac{A_{j \rightarrow i} \Lambda_{j \rightarrow i}}{\bar{v}_{-} \bar{q} n_{-}^{\mathrm{s}}}-\frac{T_{-}}{\bar{v}_{-} \bar{q} n_{-}^{\mathrm{s}} n_{0}}=0
\end{aligned}
$$

where the ambipolar diffusion rate is given by the order of magnitude relation

$$
\Gamma_{-}=0\left[\frac{D_{a} n_{-}^{\mathrm{s}}}{l_{-}^{2}} \bar{N}_{-}\right]
$$

$D_{a} \cong \sqrt{\pi k T_{\sigma} / 2 m_{\sigma}} \bar{\lambda}_{+}\left(1+T_{-} / T_{\sigma}\right)$ is the ambipolar diffusion coefficient. $\bar{\lambda}_{+}$is the mean free path of the ions. $l_{-}$denotes the characteristic length for spatial inhomogeneities of the macroscopic electron parameters.

The index $i_{\mathrm{T}}$ signifies the lowest atomic energy level, which within a prescribed accuracy is in Boltzmann-Saha equilibrium with all higher levels and the free electrons, i. e. for $i \geqq i_{\mathrm{T}}$ the relation $\alpha_{i} \cong \bar{N}_{-}^{2}$ holds.

c) electron energy balance

where

$$
\frac{\bar{N}_{-} n_{-}^{\mathrm{S}}(e E)^{2}}{m_{-} \sum_{\sigma \neq-} v_{-\sigma}}=\bar{N}_{-} n_{-}^{\mathrm{s}} \sum_{\sigma \neq-} v_{-\sigma} 3 \frac{m_{-}}{m_{\sigma}} k\left(T_{-}-T_{\sigma}\right)+Q_{\mathrm{R}}+\sum_{\sigma=1,+} k T_{-} \bar{\eta}_{\sigma} T_{\sigma}+\nabla \boldsymbol{q}_{-}
$$

is the average momentum transfer collision frequency. In Eq. (7) the average energy loss due to collisional energy transfer between electrons and heavy particles has been formulated on the assumption of a Maxwellian electron distribution in the low energy range. $Q_{\mathrm{R}}$ denotes the radiation loss resulting from line and continuum radiation escape and can be calculated by the formula

$$
\begin{aligned}
Q_{\mathrm{R}} & =\left[\sum_{j<i<i_{\mathrm{T}}} \sum_{i}+\bar{N}_{-}^{2} \sum_{j<i_{\mathrm{T}} \leqq i} \sum_{i} n_{i}{ }^{\mathrm{B}} k T_{-}\left(\bar{\eta}_{i}-\bar{\eta}_{j}\right) A_{i \rightarrow j} \Lambda_{i \rightarrow j}\right. \\
& +\left(\bar{N}_{-} n_{-}^{\mathrm{S}}\right)^{2} k T_{-} \bar{v}_{-} \sum_{i<i_{\mathrm{T}}} \int_{0}^{\infty} \eta^{2} \sigma_{v i}^{-+}\left(\eta, \bar{\eta}_{+}-\bar{\eta}_{i}\right) \exp (-\eta) \mathrm{d} \eta
\end{aligned}
$$

where for the adopted plasma model $\Lambda_{i \rightarrow j} \neq 0=1$ holds.

The last two terms of Eq. (5) denote the electron energy losses due to diffusion and electron heat flux. The latter is taken into account by the order of magnitude relation

$$
\left\ulcorner\boldsymbol{q}_{-}=0\left[\frac{5}{2} \frac{\bar{N}_{-} n_{-}^{\mathrm{S}}}{m_{-} \sum_{\sigma \neq-} v_{-\sigma}}\left(\frac{k T_{-}}{l_{-}}\right)^{2}\right] .\right.
$$

\section{Cross Sections for Inelastic Collisional- Radiative Processes in Cesium}

Limiting the consideration to optically allowed transitions we approximate the electron collisional excitation cross sections in the threshold region by the formula 
$Q_{-}^{-j}\left(\eta, \bar{\eta}_{j}-\bar{\eta}_{i}\right)=S_{i \rightarrow} k T_{-}\left(\bar{\eta}_{j}-\bar{\eta}_{i}\right)\left[1-\left(\bar{\eta}_{j}-\bar{\eta}_{i}\right) / \eta\right]$

with $\quad S_{i \rightarrow j}=0.45 \pi \bar{q} \frac{f_{i \rightarrow j}}{k T_{-}\left(\bar{\eta}_{j}-\bar{\eta}_{i}\right)^{3}}$

which follows from the distorted wave approximation ${ }^{14}$ by an appropriate choice of the effective Gaunt factor. $f_{i \rightarrow j}$ is the absorption oscillator strength for the transition $i \rightarrow j$. For the cesium atom, the experimentally determined threshold slope of the resonance excitation cross section is $S_{0 \rightarrow 1}$ $\cong 95 \AA^{2} / \mathrm{ev}^{15}$. This value is smaller than the theoretical value by a factor of approximately 0.7 (when using $f_{0 \rightarrow 1} \cong 1.13^{16}$ ).

The electron collisional ionization cross sections have been calculated according to Gryzinski's theory ${ }^{17}$, i. e.

$$
\begin{aligned}
Q_{-i}^{-\AA^{+}}\left(\eta, \bar{\eta}_{+}-\bar{\eta}_{i}\right) & \\
= & C q\left(\eta / \bar{\eta}_{+}-\bar{\eta}_{i}\right) /\left(k T_{-}\right)^{2}\left(\bar{\eta}_{+}-\bar{\eta}_{i}\right)^{2}, \text { whęre } \\
q(x) \equiv & \frac{1}{x}\left(\frac{x-1}{x+1}\right)^{3 / 2} \\
& \cdot\left[1+\frac{2}{3}\left(1-\frac{1}{2 x}\right) \ln (2.7+\sqrt{x-1})\right] \quad(11)
\end{aligned}
$$

$C$ is a constant, which for cesium amounts to $6.56 \cdot 10^{2} \AA^{2}(\mathrm{ev})^{2}$.

For ionization from the ground state a cross section formula for the threshold region analogeous to that for excitation may be used, i. e.

$$
Q_{-_{0}^{-+}}^{\overline{-}^{-+}}\left(\eta, \bar{\eta}_{+}\right)=S_{0 \rightarrow+} k T_{-} \bar{\eta}_{+}\left(1-\bar{\eta}_{+} / \eta\right)
$$

where for cesium the value $S_{0 \rightarrow_{+}} \cong 2.7 \AA^{2} / \mathrm{ev}$ can be deduced from experimental data ${ }^{18,19}$.

The radiative recombination cross sections for the lowest atomic energy levels have been used in the simple form

$$
\sigma_{\nu i}^{\nu_{i}^{+}}\left(\eta, \bar{\eta}_{+}-\bar{\eta}_{i}\right)=c_{i} / k T_{-} \eta
$$

which agrees with theoretical predictions ${ }^{20,21}$ and experimental findings ${ }^{22,23}$ concerning the threshold behaviour of the recombination cross sections in cesium. The values of the constants $c_{i}$ have been calculated by comparison with the theoretical data.

\section{Resonance Radiation Escape Factor}

The escape factor for resonance radiation in low temperature cesium plasmas with moderate ionization degrees is essentially determined by resonance line broadening. For a spatially homogeneous plane parallel plasma slab of infinite lateral extent the escape factor for line radiation with a Lorentzian line profile can be analytically represented in terms of the expression ${ }^{24}$

$$
\begin{aligned}
& \frac{1}{2} \int_{1}^{\infty} \frac{T_{j \rightarrow a}(\tau \Delta)}{\tau^{2}} \mathrm{~d} \tau \\
& \text { with } T_{j \rightarrow i}(\tau \Delta)=I_{0}\left(\frac{x_{i \rightarrow j} \tau \Delta}{\pi \delta v}\right) \exp \left\{\frac{-x_{i \rightarrow j} \tau \Delta}{\pi \delta v}\right\}
\end{aligned}
$$

where $\varkappa_{i \rightarrow i}$ and $\delta v$ are respectively the reabsorption coefficient in the line center and the line width. $I_{0}$ denotes the modified Bessel function of zero order. Estimates on the basis of Eq. (14) show that for cesium plasmas of linear dimensions $\Delta$ between $10^{-3} \mathrm{~m}$ and $10^{-1} \mathrm{~m}$ the resonance radiation escape factor varies from some $10^{-3}$ down to some $10^{-4}$.

\section{Solution Method}

The solution of the kinetic equation for the isotropic part of the electron distribution function proceeds as described in the previous paper by subdividing the electron energy range into multiples of the resonance excitation energy and using the inequality

$$
\left(1+\frac{\bar{\eta}_{1}}{\eta}\right) \frac{Q_{-0}^{-1}\left(\eta+\bar{\eta}_{1}, \bar{\eta}_{1}\right)}{Q_{-0}^{-1}\left(\eta, \bar{\eta}_{1}\right)} \exp \left(-\bar{\eta}_{1}\right) \ll 1
$$

for all but the first subregion $\left(0 \leqq \eta \leqq \bar{\eta}_{1}\right)$. The appearance of a term proportional to $\gamma\left(\eta+\bar{\eta}_{1}\right)$ in the kinetic equation for the first subregion suggests an iterative solution procedure. The lowest order of the iterative scheme, $\gamma^{(0)}(\eta)$, is calculated by completely ignoring inelastic and superelastic electron collisions in the first subregion. The first order approximation, $\gamma^{(1)}(\eta)$, is then determined by substitution of $\gamma^{(0)}(\eta)$ in the inelastic and superelastic collision terms of the first subregion. The continuation of this iteration leads to a rapid convergence of the solution, since inelastic and superelastic collision processes do not very much disturb the electron distribution in the thermal energy range, provided that $\exp \left(-\bar{\eta}_{1}\right) \ll 1$.

Substitution of Eq. (10) for the collisional excitation cross section of the resonance level yields an analytical solution, which in the lowest order of approximation and for the first two energy subregions reads 


$$
\begin{aligned}
\gamma_{\mathrm{I}}^{(0)}(\eta) & =1+K_{1}(\exp \eta-1) \text { for } 0 \leqq \eta \leqq \bar{\eta}_{1}, \\
\gamma_{\mathrm{II}}{ }^{(0)}(\eta) & =\exp \frac{\eta}{2}\left[K_{2} \operatorname{Ai}(\xi)+K_{3} \operatorname{Bi}(\xi)\right] \\
& +\alpha_{1} \gamma_{\mathrm{I}}^{(0)}\left(\eta-\bar{\eta}_{1}\right) \text { for } \quad \bar{\eta}_{1} \leqq \eta \leqq 2 \bar{\eta}_{1} .
\end{aligned}
$$

$\operatorname{Ai}(\xi)$ and $\operatorname{Bi}(\xi)$ are Airy functions ${ }^{25}$ of the argument $\xi=\xi_{1}+\varkappa_{1}^{1 / 3}\left(\eta-\bar{\eta}_{1}\right)$ with $1 / \xi_{1}=4 \varkappa_{1}{ }^{2 / 3}$, where

$$
\varkappa_{1}=n_{0} S_{0 \rightarrow 1} k T_{-} \bar{\eta}_{1} / v_{--}^{*}
$$

is the ratio between the inelastic electron-atom col- lision rate and the electron-electron interaction rate. The integration constants $K_{1} \ldots K_{3}$ follow from the normalization condition for the electron distribution function, which implies a finite value of $\gamma(\eta)$ for $\eta \rightarrow \infty$, and the continuity condition for the electron distribution function and its derivative. In particular, we have

$$
\sum_{k} a_{i k} K_{k}=b_{i}
$$

where

$$
\begin{aligned}
& a_{1 k} \cong\left(\begin{array}{c}
\exp \bar{\eta}_{1} \\
-\exp \frac{\bar{\eta}_{1}}{2} \operatorname{Ai}\left(\xi_{1}\right) \\
-\exp \frac{\bar{\eta}_{1}}{2} \operatorname{Bi}\left(\xi_{1}\right)
\end{array}\right) \quad a_{2 k} \cong\left(\begin{array}{c}
\exp \frac{\bar{\eta}_{1}}{2} \\
-\frac{1}{2} \mathrm{Ai}\left(\xi_{1}\right)-\varkappa_{1}^{1 / 3} \mathrm{Ai}^{\prime}\left(\xi_{1}\right) \\
-\frac{1}{2} \mathrm{Bi}\left(\xi_{1}\right)-\varkappa_{1}^{1 / 3} \mathrm{Bi}^{\prime}\left(\xi_{1}\right)
\end{array}\right) \\
& a_{3 k} \cong\left(\begin{array}{c}
\alpha_{1}\left[\frac{1}{2}-\varkappa_{1}^{1 / 3} \frac{\operatorname{Ai}^{\prime}\left(\xi_{2}\right)}{\operatorname{Ai}\left(\xi_{2}\right)}\right] \\
-\frac{\alpha_{1} \varkappa_{1}^{1 / 3}}{\operatorname{Ai}\left(\xi_{2}\right)} \exp \left(-\frac{\bar{\eta}_{1}}{2}\right) \int_{\xi_{2}}^{\xi_{3}} \operatorname{Ai}(\tau)\left(\tau-\xi_{1}\right) \operatorname{Ai}\left(\tau-\varkappa_{1}^{1 / 3} \bar{\eta}_{1}\right) \mathrm{d} \tau \\
\frac{\varkappa_{1}^{1 / 3}}{\pi \operatorname{Ai}\left(\xi_{2}\right)}\left[1-\alpha_{1} \pi \exp \left(-\frac{\bar{\eta}_{1}}{2}\right) \int_{\xi_{2}}^{\xi_{3}} \operatorname{Ai}(\tau)\left(\tau-\xi_{1}\right) \operatorname{Bi}\left(\tau-\varkappa_{1}^{1 / 3} \bar{\eta}_{1}\right) \mathrm{d} \tau\right]
\end{array}\right), \quad \xi_{n}=\xi_{1}+(n-1) \varkappa_{1}^{1 / 3} \bar{\eta}_{1},
\end{aligned}
$$

and

$$
b_{i} \cong\left(\begin{array}{c}
\alpha_{1}-1 \\
0 \\
\frac{\alpha_{1}\left(1-\alpha_{1}\right)}{\exp \bar{\eta}_{1}}\left[\frac{1}{2}+\varkappa_{1}^{1 / 3} \frac{\operatorname{Ai}^{\prime}\left(\xi_{2}\right)}{\operatorname{Ai}\left(\xi_{2}\right)}\right]
\end{array}\right) .
$$

The expressions for the matrix and vector elements $a_{i k}$ and $b_{i}$ are approximate in the sense that the conditions

$$
\begin{array}{r}
\alpha_{1} \exp \left(-\bar{\eta}_{1}\right) \ll 1, \alpha_{1} \exp \left(-\frac{\bar{\eta}_{1}}{2}\right) \frac{\operatorname{Ai}\left(\xi_{3}\right)}{\operatorname{Ai}\left(\xi_{2}\right)} \ll 1, \\
\text { and } \alpha_{1} \exp \left(-\frac{\bar{\eta}_{1}}{2}\right) \frac{\operatorname{Ai}^{\prime}\left(\xi_{3}\right)}{\operatorname{Ai}^{\prime}\left(\xi_{2}\right)} \ll 1
\end{array}
$$

must be satisfied.

As demonstrated in the previous paper, a simple recursion formula

$$
\gamma(\eta) \cong \alpha_{1} \gamma\left(\eta-\bar{\eta}_{1}\right)
$$

exists for all values $\eta$, for which $\varkappa_{1}\left(\eta-\bar{\eta}_{1}\right) \gg 1$ holds. Since for low electron temperatures the recursion formula is usually valid for $\eta \geqq 2 \bar{\eta}_{1}$, it is sufficient to calculate the electron distribution function within the first two energy subregions, according to Equation (16).

Substitution of the analytical solution, Eq. (16), into the collision integrals of the particle balance equations and application of the recursion formula, Eq. (20), leads to a system of nonlinear coupled equations for $\alpha_{i}$ and $\bar{N}_{-}$, which are numerically solved by an iteration method.

\section{Results and Discussion}

The nonequilibrium population densities of bound and free electron states have been numerically evaluated for the example of a cesium plasma. The calculations were performed for a prescribed 
number density $n_{0}=10^{15} \mathrm{~cm}^{-3}$ of ground state atoms and a translational temperature $T_{\sigma}=10^{3} \mathrm{~K}$ of the heavy particles taking into account up to 41 atomic energy levels, 25 of which were assumed to be in a nonequilibrium state. The parameters, which determine the departure from equilibrium, are the radiation escape factors and the characteristic $n . \mathrm{cm}^{-3}$ lengths for excitation diffusion and ambipolar diffusion. In particular, it is assumed that nonresonance radiation freely escapes the plasma volume and nonresonance level diffusion is negligibly small, i. e. that $\Lambda_{i \rightarrow j \neq 0}=1$ and $l_{i>1}=\infty$ hold.

In Fig. 1 we first compare the results $n_{-}\left(T_{-}\right)$ as calculated with the distorted electron distribution function (solid lines) with those derived on the assumption of a Maxwellian electron distribution (dashed lines). The accuracy of the results as determined by the number of considered atomic energy levels is indicated by the values $\alpha_{i т} / \bar{N}_{-}^{2}$. Values $\alpha_{i T} / \bar{N}_{-}^{2}<1$ indicate an increase, values $\alpha_{i T} / \bar{N}_{-}^{2}>1$ a decrease of the electron density with increasing number of atomic energy levels under consideration. The calculations show that the number of atomic energy levels used in our model becomes insufficient towards the lower branches of the $n_{-}\left(T_{-}\right)$ diagrams. The results are demonstrated both without and with consideration of resonance level diffusion and ambipolar diffusion (plots I and II, III). For all cases the resonance radiation escape factor is assumed to be $\Lambda_{1 \rightarrow 0}=10^{-3}$. The characteristic lengths for resonance level diffusion and ambipolar diffusion are taken to be equal and are supposed to lie within the centimeter range, where small variations in the length scale induce large changes in the departure from Saha equilibrium, as can be seen by comparison between plots II and III. One observes that at a fixed electron density increased plasma losses by additional diffusion processes (or increased radiation escape) weaken the Maxwellization process through an electron temperature elevation, whereas at fixed electron temperature increased plasma losses enhance the Maxwellization rate in the lower branches of the $n_{-}\left(T_{-}\right)$plots through an electron density elevation. Generally, the difference between solid and dashed lines increases with decreasing electron density and increasing electron temperature. This is, of course, a consequence of the lowering of the Coulomb cross section determining the parameter $\varkappa_{1}$, which in turn together with a lowering of $\alpha_{1}$ favours the disturbance of the electron distribution.

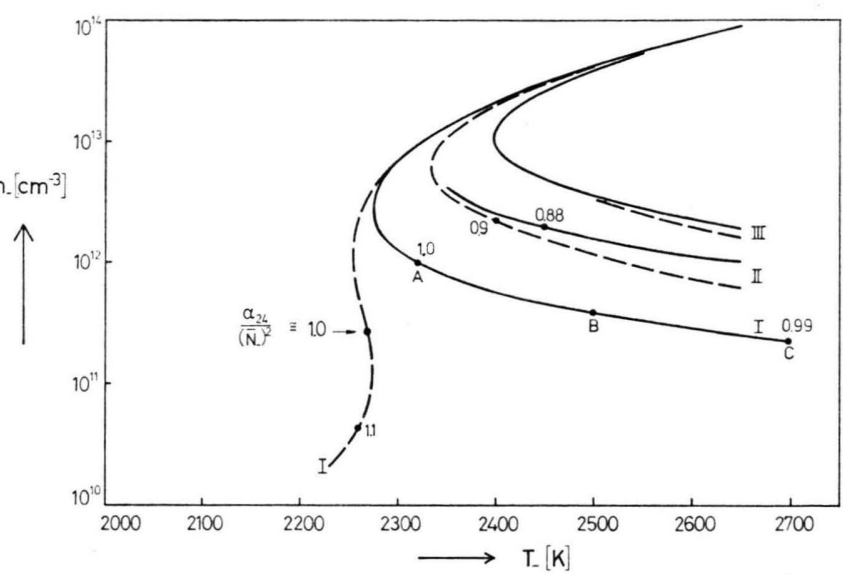

Fig. 1. Deviations from Saha equilibrium, --- on the assumption of a Maxwellian electron distribution, taking into account deviations from the Maxwellian electron distribution, parameter values:

$$
\begin{gathered}
n_{0}=10^{15} \mathrm{~cm}^{-3}, T_{\sigma}=10^{3} \mathrm{~K}, \\
\Lambda_{1 \rightarrow 0}=10^{-3}, \quad \Lambda_{i \rightarrow j \neq 0}=1, l i>1=\infty, \\
l_{1}=l_{-}=\infty \text { (I), } 2 \mathrm{~cm}(\mathrm{II}), 1 \mathrm{~cm}(\mathrm{III}) .
\end{gathered}
$$

The decrease of the Maxwellization rate by lowering the electron density and increasing the electron temperature is clearly seen from Figure 2. In this figure the normalized electron distribution function $\gamma(\eta)$ is shown for three different points (marked A, B, and C) on the lower branch of plot I, Figure 1 . For $\eta \geqq 2 \bar{\eta}_{1}$ the results calculated with the recursion formula (dashed lines) very well agree with those derived from the exact analytical formulae (solid lines).

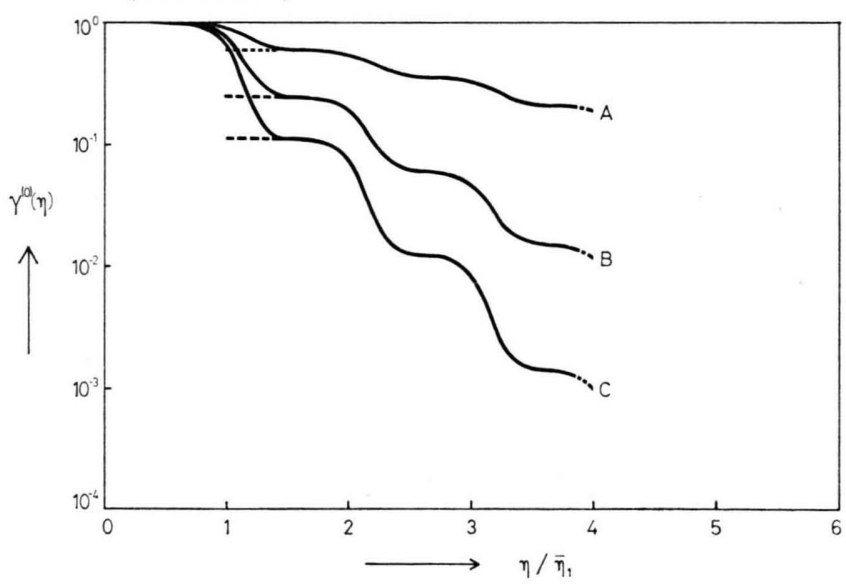

Fig. 2. Deviations from the Maxwellian electron distribution for the points $\mathrm{A}, \mathrm{B}$, and $\mathrm{C}$ of plot I, Fig. 1, - - recursion formula, exact analytical solution. 
The $n_{-}\left(T_{-}\right)$functions are multivalued above certain critical values of the electron temperature and the plasma loss parameters. This ambiguity can be explained by the cumulative ionization mechanism: for high electron densities the enhanced diffusion and radiation losses can be compensated by an enhanced cumulative ionization, whereas for low electron densities, ionization preferentially from the ground state is sufficient to balance the decreased plasma losses. Moreover, with decreasing electron density the influence of radiative recombination increases in comparison to electronic three body recombination. Generally, consideration of diffusion processes and/or deviations from the Maxwellian electron distribution leads to double valued functions $n_{-}\left(T_{-}\right)$defining a minimum electron temperature, below which the discharge is extinguished. In the diffusionfree case and for a Maxwellian electron distribution even three different values of the electron density exist for each electron temperature within the ambiguity regime. These features are, of course, embedded in the mathematical structure of the electron particle balance, Equation (5). They may be graphically deduced by plotting a normalized difference $\delta \Gamma_{-}$between ionization rate and recombination-diffusion rate as function of $\bar{N}_{-}$at fixed values of $n_{0}$ and $T_{-}$. The zeros of such a plot give the consistent solutions. Figure 3 shows, for instance, the function $\delta \Gamma_{-}\left(\bar{N}_{-}\right)$ for the diffusionfree case with $\Lambda_{1 \rightarrow 0}=10^{-3}$ at $T_{-}=2500 \mathrm{~K}$ as well as for the diffusion case with $l_{1}=l_{-}=2 \mathrm{~cm}$ at $T_{-}=2375 \mathrm{~K}$.

As already stated, for constant values of the electron density, departures from the Maxwellian electron distribution must increase the electron temperature to balance the particle production and loss rates. This, in turn, lowers the line radiation loss, as can be deduced from Eq. (8), but slightly increases the recombination radiation loss and the collisional energy transfer of the electrons. If the change in the line radiation loss is the dominant effect, then the electric energy input must decrease with increasing deviations from the Maxwellian electron distribution. Since in the considered electron temperature range the electron momentum transfer collision frequency slightly decreases with increasing electron temperature, the lowering of the electric energy input is accompanied by a simultaneous lowering of the electric field strength.

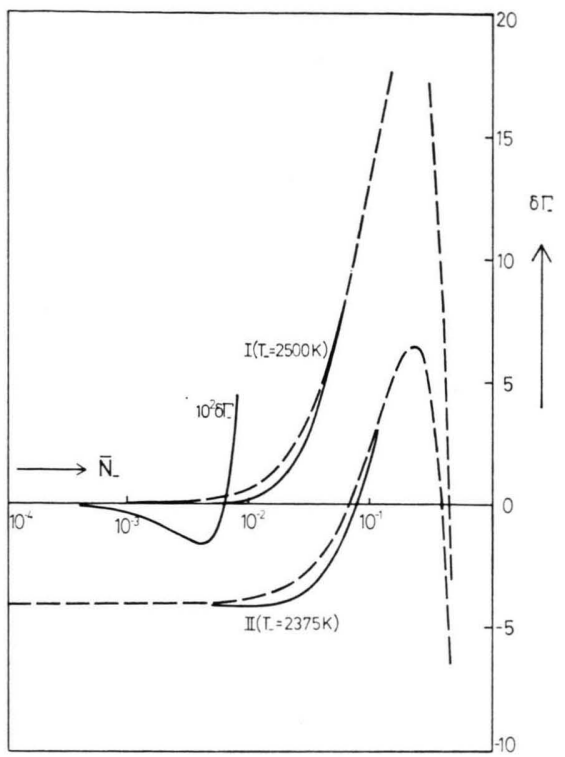

Fig. 3. Graphical solution of the electron particle balance by plotting the difference $\delta \Gamma_{-}\left(N_{-}\right)$between ionization rate and recombination-diffusion rate, calculated examples: $T_{-}=$ $2500 \mathrm{~K}$ of plot I and $T_{-}=2375 \mathrm{~K}$ of plot II, Figure 1 .

The decrease of the electric field strength under the action of a depletion in the high energy tail of the electron distribution is seen from Figure 4. The results are shown for the same parameter values as in Figure 1. As is to be expected, diffusion and heat conduction losses in addition to radiation escape increase the electric field strength.

After having finished up this work, some experimental and theoretical results ${ }^{26,27}$ concerning the electron distribution in the positive column of a cylindrical low pressure cesium discharge came to the author's knowledge. The measurements, which have been performed for $n_{0}=10^{15} \mathrm{~cm}^{-3}$ in the narrow energy range $1 \mathrm{ev} \leqq \epsilon_{-} \leqq 2.5 \mathrm{ev}$ indicate a nearly Maxwellian electron distribution for electron densities down to $n_{-} \cong 2 \cdot 10^{11} \mathrm{~cm}^{-3}$. For lower electron densities pronounced deviations from the Maxwellian electron distribution have been detected, which for very low electron densities again decrease-probably due to a simultaneous lowering of the electron temperature. Unfortunately, the uncertainty in the experimental plasma loss parameters prevents an exact comparison between theory and experiment. This is particularly so, since an additional consideration of diffusion processes can be compensated by a simultaneous reduction of the radiation escape factors. As a matter of fact, even on the assumption of vanishing diffusion, both the 


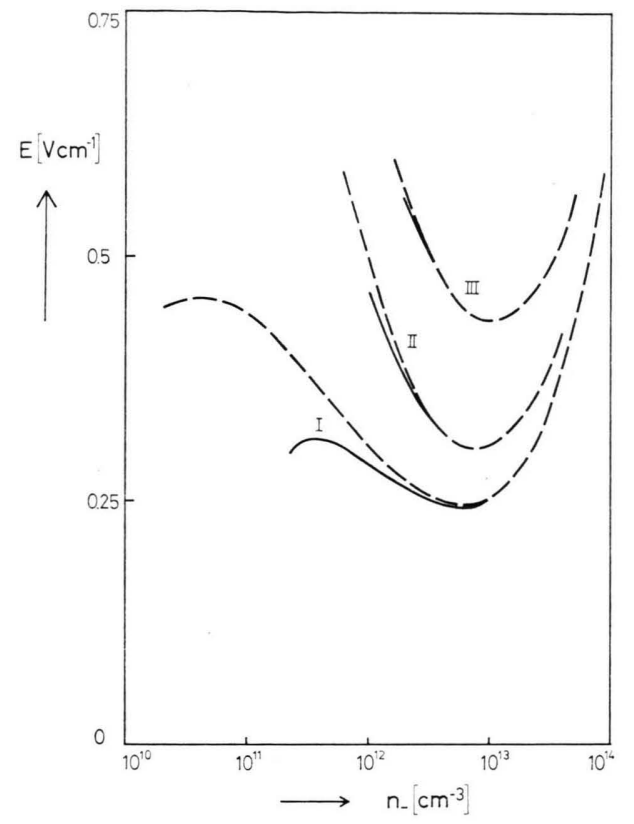

Fig. 4. Influence of deviations from the Maxwellian electron distribution on the electric field strength. Parameter values as in Figure 1. theoretical work done specifically for these experiments and our theoretical results predict deviations from the Maxwellian electron distribution for much higher electron densities than $n_{-}=2 \cdot 10^{11} \mathrm{~cm}^{-3}$, if $\Lambda_{i \rightarrow j \neq 0}=1$ and $\Lambda_{1 \rightarrow 0} \geqq 10^{-4}$.

\section{Appendix}

There are a number of effects, which are omitted from consideration in order to simplify the theoretical model. The validity and consistency of such model simplifications are established by certain criteria, which are summarized in Table 1.

In these criteria $\lambda_{-\alpha}^{\varepsilon, \mathrm{P}}(\eta)$ are respectively the energy and the momentum relaxation lengths for electrons of energy $\epsilon_{-}=k T_{-} \eta$ colliding with particles of a Maxwellized plasma species $\alpha . \tau_{i}$ and $\sigma_{\nu i}^{-+}\left(\nu, \bar{\eta}_{+}-\bar{\eta}_{i}\right)$ are the effective deexcitation time and the photo-ionization cross section of the atomic energy level $i$. Moreover, $Q_{0 i}^{0 j}\left(\zeta, \in_{j}-\epsilon_{i}\right)$ with $\zeta=$ $\sqrt{m_{\sigma} / 4 k T_{\sigma}}\left|\boldsymbol{v}_{i}-\boldsymbol{v}_{0}\right|$ denotes the cross section for

Table 1.

Neglected effects concerning the isotropic part of the electron distribution function

electron diffusion by spatial inhomogeneities and electric fields

elastic electron-heavy particle collisions

unbalanced electron collisional atomic nonresonance transitions

deviations from the Maxwellian distribution in the integrals of the Fokker-Planck term

Neglected effects concerning the atomic level population

nonresonance excitation diffusion

deviations from the Maxwellian distribution in the recombination coefficients

reabsorption of nonresonance line radiation

reabsorption of recombination radiation

inelastic atom-atom collisions validity criteria

$$
\begin{aligned}
& \frac{\lambda_{--}^{s}(\eta)}{3 \underset{\sigma \neq-}{\eta l_{-}^{2} \sum 1 / \lambda_{-\sigma}^{\underline{p}}(\eta)}} \max \left[1,\left(\frac{e E l_{-}}{k T T_{-}}\right)^{2}\right] \ll 1 \\
& \sum_{\sigma \neq-} 2 \frac{m_{-}}{m_{\sigma}} \frac{\lambda_{--}^{\mathrm{P}}(\eta)}{\lambda_{-\sigma}^{\mathrm{P}}(\eta)} \ll 1 \\
& \sum_{j i<j} \sum_{\frac{n_{i}}{\mathrm{~B}}}\left[\alpha_{i} \gamma(\eta)-\alpha_{j} \gamma\left(\eta-\bar{\eta}_{j}+\bar{\eta}_{i}\right)\right] Q_{-i}^{-3}\left(\bar{\eta}, \bar{\eta}_{j}-\bar{\eta}_{i}\right) \\
& \ll\left[\gamma(\eta)-\alpha_{1} \gamma\left(\eta-\eta_{1}\right)\right] Q_{\underline{-}_{0}^{-1}}^{-1}\left(\eta, \bar{\eta}_{1}\right)
\end{aligned}
$$

validity criteria

$\frac{D_{i} \tau_{i}}{l_{i}^{2}} \ll 1$ for $i>1$

$$
\begin{aligned}
& \exp \left(-\bar{\eta}_{1}\right) \ll 1 \\
& \frac{x_{i \rightarrow j} \Delta}{\pi \delta \nu} \ll 1 \\
& n_{i} \sigma \hat{\uparrow}_{v v}^{+}\left(\eta, \bar{\eta}_{+}-\bar{\eta}_{i}\right) \Delta \ll 1
\end{aligned}
$$

$\frac{n_{0}}{n_{-}} \sqrt{8 \frac{m_{-} T_{\sigma}}{m_{\sigma} T_{-}}} \int_{0}^{\infty} \zeta^{3} \exp \left(-\zeta^{2}\right) Q_{0 i}^{01}\left(\zeta, \epsilon_{j}-\epsilon_{i}\right) \mathrm{d} \zeta$

$\ll \int_{0}^{\infty} \eta \exp (-\eta) Q_{-i}^{-j}\left(\eta, \overline{\eta_{j}}-\bar{\eta}_{i}\right) \mathrm{d} \eta$ 
the excitation transition $i \rightarrow j$ due to inelastic collisions between excited and ground state atoms ${ }^{28}$.

The consideration of ambipolar diffusion in the electron particle balance is consistent with the neglect of electron diffusion in the kinetic equation for the isotropic part of the electron distribution, if

$$
\lambda_{--}^{\varepsilon}(\eta) / D_{a} \tau_{-} \sum_{\sigma \neq-} 1 / \lambda_{-\sigma}^{\mathrm{P}}(\eta) \ll 1
$$

holds, where $\tau_{-}$denotes the effective recombination time of the free electrons.

1 D. R. Bates, A. E. Kingston, and R. W. P. McWhirter, Proc. Phys. Soc. London A 267, 297 [1962] and A 270, 155 [1962].

2 D. W. Norcross and P. W. Stone, J. Quant. Spectrosc. Radiat. Transfer 8, 655 [1968].

3 H. W. Drawin, Z. Naturforsch. 25 a, 145 [1970].

4 B. Sayer, J. C. Jeannet, and J. Berlande, J. Physique 33, 993 [1972].

5 J. V. Dugan, Jr., F. A. Lyman, and L. U. Albers, in Electricity from MHD, Proc. of a Symp., Salzburg 4-8 July 1966, Vol. II, p. 85.

6 J. F. Shaw, M. Mitchner, and C. H. Kruger, in Electricity from MHD, Proc. of a Symp., Warsaw 24-30 July 1968, Vol. I, p. 53.

7 K. G. Saul, Phys. Fluids 14 (12), 2625 [1971].

8 L. M. Biberman, V. S. Vorob'ev, and I. T. Yakubov, High Temp. 6, 359 [1968].

${ }^{2}$ F. G. Baksht, B. Ya. Moizhes, and V. A. Nemchinskii, Sov. Phys.-Techn. Phys. 13 (10), 1401 [1969].

10 J. F. Shaw, M. Mitchner, and C. H. Kruger, Phys. Fluids $13(2), 325$ [1970].

11 D. T. Shaw, J. Appl. Phys. 42 (1), 490 [1971].

12 S. Suckewer, Z. Physik 247, 354 [1971].

13 H. A. Claaßen, Z. Naturforsch. 28 a, 1875 [1973].

14 M. J. Seaton, in Atomic and Molecular Processes (ed. D. R. Bates), Academic Press, New York 1962, p. 414.

\section{Acknowledgements}

The author would like to acknowledge Prof. G. Ecker for helpful discussions of the present work. $\mathrm{He}$ also wishes to thank Mr. H. Schlebbe, who carried out the numerical calculation. This work was performed within the activities of the Sonderforschungsbereich Plasmaphysik Bochum-Jülich.

15 I. P. Zapesochnyi and L. L. Shimon, Sov. Phys.-Doklady 11 (1), 44 [1966].

${ }^{16}$ E. M. Anderson and V. A. Zilitis, Opt. Spectrosc. 16, 211 [1964].

17 M. Gryzinski, Phys. Rev. 138, A 336 [1965].

18 K. J. Nygaard, J. Chem. Phys. 49 (5), 1955 [1968].

19 I. P. Zapesochnyi and I. S. Aleksakhin, Sov. Phys.-JETP 28 (1), 41 [1969].

20 D. W. Norcross and P. M. Stone, J. Quant. Spectrosc. Radiat. Transfer 6, 277 [1966].

21 J. C. Weisheit, J. Quant. Spectrosc. Radiat. Transfer 12, 1241 [1972].

22 L. Agnew and C. Summers, Proc. VII. Intl. Conf. on Phenomena in Ionized Gases, Belgrade 1965, p. 574.

23 S. M. Gridneva, and G. A. Kasabov, High Temp. 5, 334 [1967].

24 H. A. Claaßen, Rep. JUL-757-TP, KFA Jülich GmbH [1972].

25 M. Abramowitz and I. A. Stegun, Handbook of Mathematical Functions, Dover Publ. Inc., New York 1965.

26 A. Bourdier, Doctorial Thesis, Université de Paris Sud, Centre d'Orsay [1972].

27 Y. Archambault and A. Bourdier, C. R. Acad. Sci. Paris 276 B, 833 [1973].

28 H. A. Claaßen, Z. Naturforsch. 26 a, 1888 [1971]. 